\title{
Modelo de análisis económico para proyectos dendroenergéticos: Metodología y
}

\section{resultados preliminares.}

\author{
Sonia Varón Quinche \\ Universidad Autónoma De Colombia \\ Recibido: 15/04/2021 Revisado: 21/05/2021 Aceptado: 26/06/2021 Publicado: 28/07/2021
}

\section{Resumen}

Este trabajo recopila los resultados preliminares en la implementación de cálculos matemáticos cuyo propósito es generar indicadores de viabilidad económica para proyectos de inversión y apoyar la toma de decisiones, a partir del uso de Excel, como parte del trabajo adelantado en el semillero de investigación Bio-industrias.

Dentro del estudio previo de los antecedentes del estado del arte, se ha determinado que los proyectos de inversión para la generación de energía bajo fuentes no convencionales FNCER en Colombia presentan matices de orden jurídico, contable y financiero que inciden en su viabilidad económica, cuya estructura se distingue de los proyectos convencionales, por consiguiente, se genera la necesidad de programar la herramienta propuesta bajo estas

directrices, involucrando las tecnologías existentes y alineándolas con la normatividad jurídica y fiscal vigente.

El desarrollo plasma el montaje realizado en Excel de las operaciones matemáticas que parten de datos iniciales, suministrados por el usuario, estructurando la sintaxis necesaria para calcular los indicadores. Se concluye de manera preliminar que la metodología implementada es funcional para cualquier tipo de proyecto de inversión, dando la opción de parametrizar aquellas variables con incidencia en proyectos específicos como el desarrollado, permitiendo que esta herramienta pueda adecuarse a las necesidades específicas del usuario final.

BIOTECH \& ENGINEERING Untels. Jul - Dic.1(2), 2021; ISSN:2788 -4295; 86-110 DOI: https://doi.org/10.52248/eb.Vol1Iss02.25 
Palabras clave: Viabilidad financiera, programación, sintaxis, FNCER, indicadores. Abstract

This work compiles the preliminary results in the implementation of mathematical calculations whose purpose is to generate indicators of economic viability for investment projects and support decision-making, based on the use of Excel, as part of the work carried out in the Bio- industries. Within the previous study of the antecedents of the state of the art, it has been determined that investment projects for the generation of energy under unconventional sources FNCER in Colombia present nuances of a legal, accounting and financial nature that affect their economic viability, whose structure differs from conventional projects, therefore, the need to program the proposed tool is generated under these guidelines, involving existing technologies and aligning them with current legal and fiscal regulations.

The development reflects the assembly carried out in Excel of the mathematical operations that start from initial data, supplied by the user, structuring the necessary syntax to calculate the indicators. It is preliminary concluded that the implemented methodology is functional for any type of investment project, giving the option of parameterizing those variables that affect specific projects such as the one developed, allowing this tool to be adapted to the specific needs of the end user.

Keywords: Financial viability, programming, syntax, FNCER, indicators. 


\section{Introducción}

\section{Estado del arte}

La energía obtenida a partir de la madera se conoce como dendroenergía. Esta fuente de energía se usa de forma directa, así como en sus diferentes transformaciones, plenamente aprovechables (Patiño Diez \& Smith Quintero, 2008). Dentro de los usos más conocidos, se enumeran las siguientes:

- En el uso doméstico, se usa la leña como fuente directa de calor, siendo esta la forma más antigua de obtención de energía calorífica;

- En el uso comercial e industrial, se usan los biocombustibles para el funcionamiento de maquinaria y vehículos

- Como fuente de energía eléctrica.

Dentro de las tecnologías existentes se encuentra el ciclo orgánico de Rankine que funciona a partir de turbinas de vapor, los gasificadores, las plantas de combustión, entre otras.

La dendroenergía ha venido ganando aceptación e iniciativas de estudio para su implementación. "La utilización de la biomasa, frente a los hidrocarburos, presenta ventajas comparativas que la hacen atractiva para su utilización en procesos de generación de energía, térmica, motriz o eléctrica, bien sea en sistemas centralizados, en sistemas de generación de energía distribuida o para zonas no interconectadas (ZNI)" (AENE Consultoria S.A - UPME, 2003, pág. 11)

Cabe resaltar que la dendroenergía se ha considerado una alternativa viable para la independencia a los combustibles fósiles. Patiño Diez \& Smith Quintero (2008) afirman: “Aspectos como los anteriores, colocan la energía forestal en una perspectiva de desarrollo 
optimista en muchos países, prometiendo impactos positivos tanto en el aspecto social como económico" (p. 20).

Mercado: Desde el año 1995, la industria del papel dejó de consumir madera de los bosques, para pasar a las plantaciones forestales, sin embargo, se estima que el mercado de la madera para la construcción y usos artesanales aún tiene un $80 \%$ de su obtención de materia prima de los bosques (Minambiente, 2015).

El mercado maderero se encuentra bastante concentrado en el país. El primer productor de madera, que ocupa mas del 50\% de la demanda nacional está en Cundinamarca (Bogotá, Soacha), seguida por la región Medellín-Valle de Aburrá, Bucaramanga-Girón, Cali- Yumbo y Barranquilla (Minambiente, 2015).

Este mercado presenta una alta informalidad en los procesos de producción. El Ministerio de Ambiente (2015) indica:

"La informalidad se ocasiona fundamentalmente por la ausencia de estandares de calidad para la madera, en un gran volumen de comercio; una parte se abastece de forma ilegal, extraida de los bosques naturales y un alto porcentaje de la madera aserrada se dedica a usos de bajo valor agregado o donde la madera es desechada, por lo menos parcialmente, después de usarse" (p. 14).

Esto explica que los residuos aprovechables se encuentren dispersos en los bosques.. Al respecto, AENE Consultoria S.A - UPME (2003), comenta:

"En vista de que la industria del aserrio experimentó un descenso debido a las limitaciones ambientales establecidas para las grandes empresas, la industria maderera se ha fraccionado en un sin número de pequeñas explotaciones o empresas unipersonales e informales que explotan el bosque sin atender las restricciones legales y ambientales, siendo el intermediario el que 
comercia con la madera y abastece la industria del mueble, construcción, fabrica de chapas, triplex, etc. Estas explotaciones individuales y el uso progresivo de motosierras para procesar madera escuadrada, directamente en el bosque hace que los residuos del bosque natural provenientes del desrrame, decortezado, troce y corte queden diseminados directamente en el bosque, por tanto el potencial energético no se puede encontrar concentrado” (p. 100).

Generación de empleo: El sector maderero es uno de los mas importantes por la diversificación de productos y de actividades a desempeñar, tales como la tala y producción de pulpa. La cadena productiva es generadora de oferta laboral de personal no calificado (Mesa Sierra, 2016)

Se estima que del total de la población económicamente activa el $20 \%$ se encuentra empleada en el sector agrícola, abarcando un total de 3.7 millones de personas. De este total, se estima que del 10\% al 20\% corresponde al sector forestal: estando un total de 74.000 personas con vinculación directa, y dada la proporcion de plazas laborales de 1:3 entre empleos directos e indirectos, se estima que el país cuenta con un total de 296.000 trabajadores informales en el sector (Mesa Sierra, 2016). En zonas ZNI, la mano de obra requerida en buena parte es no calificada (AENE Consultoria S.A - UPME, 2003) ${ }^{1}$

Respecto al recurso disponible, la nación cuenta con recursos suficientes para generar energía de forma sostenible. Sin embargo, determinados conflictos subyacentes que guardan relación con la expansión de otras actividades económicas han ocasionado que las zonas protegidas se vean afectadas por conflictos asociados a la tenencia de la tierra (AENE Consultoria S.A - UPME, 2003). Esto genera un rezago en el aprovechamiento del mercado forestal. 
Respecto a la inversión, al encontrarse la nación en una etapa temprana de implementación, motivo por el cual, y tal como se destacó en el estudio de Quintero Gonzalez \& Quintero González (2015), los costos de inversión inicial en capital y mantenimiento son bastante elevados, respecto a otras tecnologías.

Información estadística: Los proyectos tienen limitantes en la información disponible. A pesar que las autoridades ambientales han hecho esfuerzos para obtener un sostén estadístico sólido, actualmente no se cuenta con una base de datos unificada de lo siguiente:

- Materias primas y procesos asociados

- Actores involucrados

- Costos y tratamiento especifico a nivel cambiaria, contable,y jurídico.

Esto denota un problema de segmentación. Como ejemplo de esto se realizó el ejercicio de busqueda de los incentivos tributarios en la implementación de dichos proyectos y que tienen incidencia en la utilidad, asi como el procedimiento para computarlos bajo la norma fiscal (ley 1715 de 2014). La información se encontraba dispersa en varios decretos, tomando como ejemplo el incentivo de depreciación acelerada, en el cual solamente se indica el porcentaje anual máximo permitido. Esto implica una mayor gasto en recursos. Al respecto, en el documento "Uso y Legalidad de la madera " publicado por Minambiente en 2015, se indica:

"Los resultados coinciden en identificar caracteristicas del sector, que se han mantenido en el tiempo, convirtiendose en limitantes para el manejo sostenible del bosque natural. Por ejemplo, los estudios confirman que las especies utilizadas no han cambiado, salvo las que han entrado a reemplazar a las agotadas, se continua con el aprovechamiento selectivo y el exceso de intermediarios en la comercialización, hay deficiencias en la información confiable, falta de control, la insuficiencia en los sitios de acopio se mantiene, continuan la 
falta de claridad y dificultades para el trámite de autorizaciones, y no existe o es escasa la capacitación en el sector. (Minambiente, 2015, pág. 12)

En esta publicación se midió el nivel de conocimiento en el orden nacional en cuanto a manejo sostenible de los bosques, obteniendo las siguientes puntuaciones:

\begin{tabular}{|l|c|}
\hline \multicolumn{1}{|c|}{ Aspecto } & Nivel* \\
\hline Conoce qué es manejo sostenible de bosques & 2,9 \\
\hline Considera que el manejo sostenible de los bosques permite obtener maderas legoles en el país & 3,2 \\
\hline Sabe qué es el Pacco para el uso de la madera legal & 2,2 \\
\hline Conoce las sanciones e implicaciones legales que fiene la compra y/o transporte de madera ilegal & 2,9 \\
\hline
\end{tabular}

"Escola de volores de likert: muvy olto (5), alto (4), medio (3), bcio (2), nulo (1)

Figura 1: Resultado encuestas de actores en el orden nacional. Recuperado de https://www.minambiente.gov.co/images/Bosques_BiodiversidadyServiciosEcosistemicos/pdf/Gobernanza_forestal_2/12. Uso_y_Legalidad_de_la_Madera.pdf

Este resultado indica que los encuestados tienen un limitado conocimiento sobre el tema, factor que puede incidir en el aprovechamiento eficiente del recurso.

Al respecto, Minambiente (2015) afirma que:

“Estas dificultades son ya reconocidas por el subsector forestal, que ponen en evidencia la problemática del mercado de la madera en el orden nacional, la cual constituye un factor influyente en el desarrollo social dado que conlleva a los involucrados a la busqueda de alternativas que repercutan en la dinamica del bosque y la sostenibilidad ambiental (p. 24). 


\begin{tabular}{|c|c|r|r|r|r|}
\hline Año & $\begin{array}{c}\text { Madera moviliza- } \\
\text { da bosque natural } \\
\end{array}{ }^{*\left(\mathbf{m}^{3}\right)}$ & $\begin{array}{c}\text { Madera moviliza- } \\
\text { da plantaciones } \\
{ }^{* *}\left(\mathbf{m}^{3}\right)\end{array}$ & $\begin{array}{c}\text { Importación de } \\
\text { madera sólida } \\
{ }^{* * *}\left(\mathbf{m}^{3}\right)\end{array}$ & $\begin{array}{c}\text { Importación de } \\
\text { tableros } \\
{ }^{* * *}\left(\mathbf{m}^{3}\right)\end{array}$ & $\begin{array}{c}\text { Total oferta } \\
\left(\mathbf{m}^{3}\right)\end{array}$ \\
\hline 2009 & $1.533 .653,88$ & $292.400,60$ & $18.202,61$ & $188.197,61$ & $2.032 .454,70$ \\
\hline 2010 & $1.379 .542,31$ & $1.089 .500,53$ & $35.787,24$ & $254.283,34$ & $2.759 .113,42$ \\
\hline 2011 & $1.143 .186,27$ & $753.532,06$ & $33.105,93$ & $289.631,09$ & $2.219 .455,36$ \\
\hline 2012 & $763.096,64$ & $826.860,05$ & $52.903,65$ & $317.742,25$ & $1.960 .602,59$ \\
\hline 2013 & $480.504,29$ & $1.495 .647,25$ & $34.623,42$ & $360.955,11$ & $2.371 .730,07$ \\
\hline
\end{tabular}

\begin{tabular}{|c|c|c|r|r|}
\hline Año & $\begin{array}{c}\text { Total oferta } \\
\left(\mathbf{m}^{3}\right)\end{array}$ & $\begin{array}{c}\text { Total consumo } \\
\left(\mathbf{m}^{3}\right)\end{array}$ & $\begin{array}{c}\text { Balance } \\
\left(\mathbf{m}^{3}\right)\end{array}$ & $\begin{array}{c}\text { Subregistro } \\
\left(\mathbf{m}^{3}\right)\end{array}$ \\
\hline 2009 & $2.032 .454,70$ & $3.136 .081,91$ & $-1.103 .627,22$ & \\
\hline 2010 & $2.759 .113,42$ & $3.325 .496,16$ & $-566.382,74$ & \\
\hline 2011 & $2.219 .455,36$ & $3.478 .551,48$ & $-1.259 .096,13$ & \\
\hline 2012 & $1.960 .602,59$ & $3.603 .018,47$ & $-1.642 .415,88$ & \\
\hline 2013 & $2.371 .730,07$ & $4.479 .878,28$ & $-2.108 .148,21$ & $-6.679 .670,16$ \\
\hline
\end{tabular}

Figura 2: Oferta de madera 2009-2013 y balance de oferta y demanda. Recuperado de

https://www.minambiente.gov.co/images/BosquesBiodiversidadyServiciosEcosistemicos/pdf/Gobernanza_forestal_2/12._Uso_

y_Legalidad_de_la_Madera.pdf

BIOTECH \& ENGINEERING Untels. Jul - Dic.1(2), 2021; ISSN:2788 -4295; 86-110 


\section{Planteamiento del problema}

Los proyectos de inversión para generación de energia bajo fuentes no convencionales en Colombia presentan componentes diferenciales de orden jurídico, fiscal y contable que precisan un tratamiento específico para su correcta formulación y análisis, respecto a los demás proyectos de inversión, como por ejemplo:

- Incentivos tributarios por inversión en FNCER;

- Estructura de costos (costo nivelado)

- Depreciación acelerada de activos

A la fecha, no se cuenta con una metodologia estructurada que agrupe estos factores, los cuales inciden en la utilidad final y en el concepto de viabilidad económica del proyecto de inversión, por lo que se genera la necesidad de estructurar una herramienta que incorpore estas variables, con su respectivo proceso matemático, para generar indicadores financieros acordes con la realidad económica de estos proyectos de inversión que permitan la adecuada toma de decisiones. 


\section{Desarrollo}

\section{Modelo conceptual}

La metodologia implementada consiste en la generaciòn de variables que parten de entradas suministradas por el cliente, cuyo resultado final es el cálculo de indicadores financieros.

Para definir la sintaxis se definieron las operaciones matemáticas necesarias para generar variables utilizadas en los distintos modulos bajo los cuales se generará el estado de resultados, ordenados así:

- INVERSION INICIAL

- 1. Mercado

- 2. Ingresos, ventas

- $\quad$ 3,1 Costo Nivelado

- $\quad 3.2$ Costos y Gastos

- 4. Nómina

- 5. Financiación

- INDICADORES FINALES

Se expondrán los módulos con las operaciones como paso necesario para entender su estructura, dinámica y pasos lógicos a seguir al resultado final, como objetivo principal del presente trabajo. 


\section{A. INVERSIÓN INICIAL}

En el desarrollo del módulo se tomó como punto de referencia el costo de capital estimado de una planta de biomasa con la tecnologìa $\mathrm{ORC}$, con capacidad de generación de $2 \mathrm{MW}$ :

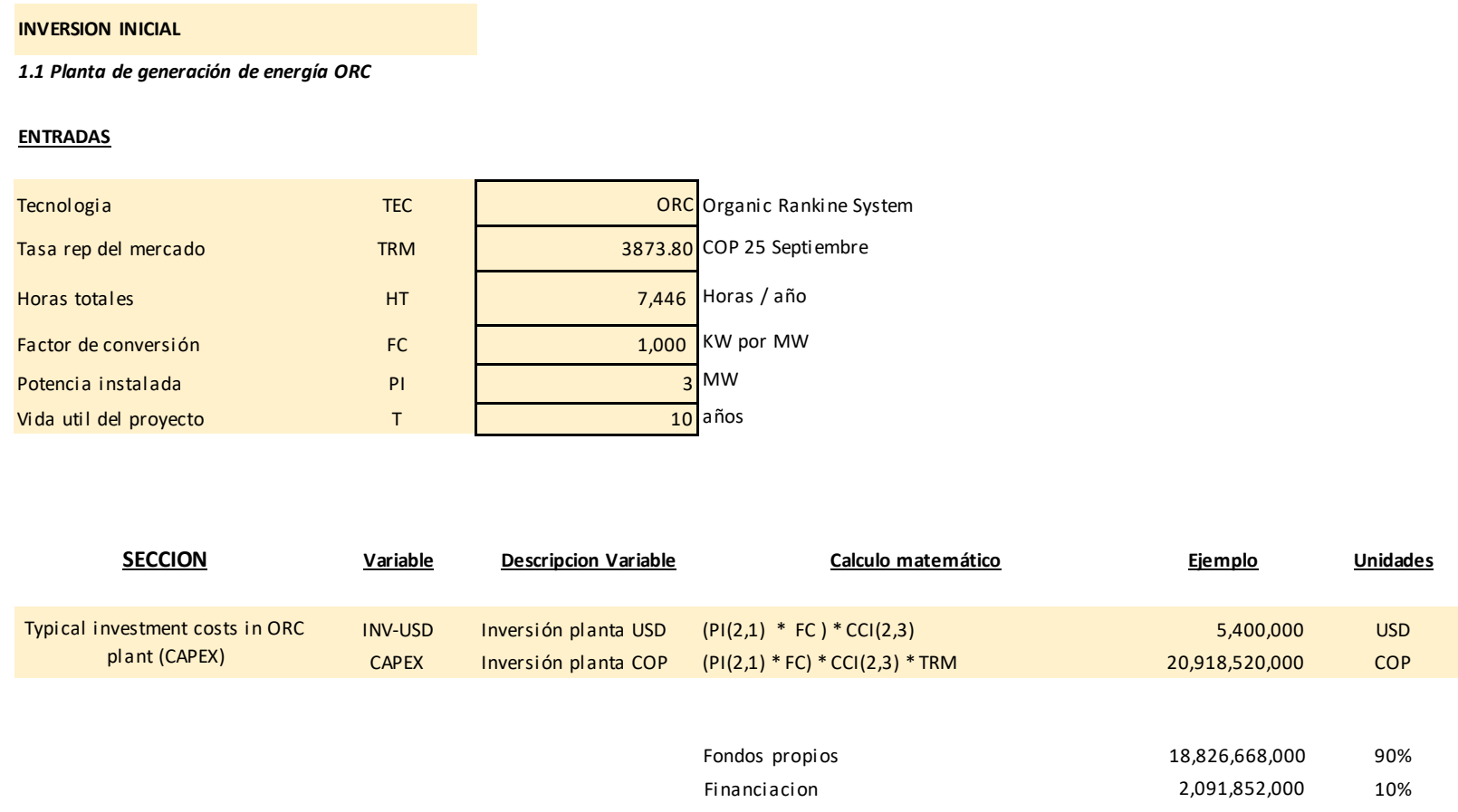

Figura 3: Mòdulo de inversión. Elaboración propia

Se han definido las tecnologìas disponibles a evaluar, así como el costo de inversión acorde con su capacidad de generación, con la variable "TEC" que es dicotómica, la cual trae al módulo los valores asociados de la tecnología escogida.

El usuario ingresa las entradas de TRM, horas totales de generación, factor de conversión, potencia y vida util del proyecto.

Se expone la presente tabla de calculo con el costo de inversión en dólares y pesos, variables definidas como INV-USD y CAPEX, sobre las cuales el usuario escoge la potencia: 


\begin{tabular}{|c|c|c|c|}
\hline & POTENCIA & Costo especifico USD / KW & \\
\hline & 1-5 MW & 1,800 & \\
\hline & 5-20 MW & 1,500 & \\
\hline & $>20 \mathrm{MW}$ & 1,200 & \\
\hline PI & $\mathrm{CCl}$ & INV-USD & CAPEX \\
\hline Potencia (MW) & Capital instalado (USD / KW) & Costo planta (USD) & Costo planta (COP) \\
\hline 1 & 1,800 & $\$ 1,800,000$ & $\$ 6,972,840,000$ \\
\hline 2 & 1,800 & $\$ 3,600,000$ & $\$ 13,945,680,000$ \\
\hline 3 & 1,800 & $\$ 5,400,000$ & $\$ 20,918,520,000$ \\
\hline 4 & 1,800 & $\$ 7,200,000$ & $\$ 27,891,360,000$ \\
\hline 5 & 1,800 & $\$ 9,000,000$ & $\$ 34,864,200,000$ \\
\hline 6 & 1,500 & $\$ 9,000,000$ & $\$ 34,864,200,000$ \\
\hline 7 & 1,500 & $\$ 10,500,000$ & $\$ 40,674,900,000$ \\
\hline 8 & 1,500 & $\$ 12,000,000$ & $\$ 46,485,600,000$ \\
\hline 9 & 1,500 & $\$ 13,500,000$ & $\$ 52,296,300,000$ \\
\hline 10 & 1,500 & $\$ 15,000,000$ & $\$ 58,107,000,000$ \\
\hline 11 & 1,500 & $\$ 16,500,000$ & $\$ 63,917,700,000$ \\
\hline 12 & 1,500 & $\$ 18,000,000$ & $\$ 69,728,400,000$ \\
\hline 14 & 1,500 & $\$ 21,000,000$ & $\$ 81,349,800,000$ \\
\hline 15 & 1,500 & $\$ 22,500,000$ & $\$ 87,160,500,000$ \\
\hline 16 & 1,500 & $\$ 24,000,000$ & $\$ 92,971,200,000$ \\
\hline 20 & 1,200 & $\$ 24,000,000$ & $\$ 92,971,200,000$ \\
\hline 21 & 1,200 & $\$ 25,200,000$ & $\$ 97,619,760,000$ \\
\hline 22 & 1,200 & $\$ 26,400,000$ & $\$ 102,268,320,000$ \\
\hline 25 & 1,200 & $\$ 30,000,000$ & $\$ 116,214,000,000$ \\
\hline
\end{tabular}

Figura 4: Costos de capital CAPEX. Elaboración propia

El cálculo matemático con el vector involucra las entradas iniciales. El costo de capital se calculó con base en el estudio realizado por ICF International, sobre los procesos WHP (waste heat to power) (ICF INTERNATIONAL, 2015): 
Table 5. Waste Heat to Power Costs

\begin{tabular}{|c|c|c|c|c|c|c|}
\hline \multirow{2}{*}{ Technology } & \multirow{2}{*}{$\begin{array}{c}\text { Cost } \\
\text { Characteristic }\end{array}$} & \multicolumn{5}{|c|}{ Electric Capacity for WHP Technology } \\
\cline { 3 - 7 } & $50-500 \mathrm{~kW}$ & $500-1,000 \mathrm{~kW}$ & $1-5 \mathrm{MW}$ & $5-20 \mathrm{MW}$ & $>20 \mathrm{MW}$ \\
\hline \multirow{2}{*}{$\begin{array}{c}\text { Steam } \\
\text { Rankine } \\
\text { Cycle }\end{array}$} & $\begin{array}{c}\text { Installed Capital } \\
\text { Cost, } \$ / \mathrm{kW}\end{array}$ & $\$ 3,000$ & $\$ 2,500$ & $\$ 1,800$ & $\$ 1,500$ & $\$ 1,200$ \\
\cline { 2 - 7 } & $\begin{array}{c}\text { O\&M Costs, } \\
\$ / \mathrm{kWh}\end{array}$ & $\$ 0.013$ & $\$ 0.009$ & $\$ 0.008$ & $\$ 0.006$ & $\$ 0.005$ \\
\hline \multirow{2}{*}{$\begin{array}{c}\text { Organic } \\
\text { Rankine } \\
\text { Cycle }\end{array}$} & $\begin{array}{c}\text { Installed Capital } \\
\text { Cost, \$/kW }\end{array}$ & $\$ 4,500$ & $\$ 4,000$ & $\$ 3,000$ & $\$ 2,500$ & $\$ 2,100$ \\
\cline { 2 - 7 } & $\begin{array}{c}\text { O\&M Costs, } \\
\$ / \mathrm{kWh}\end{array}$ & $\$ 0.020$ & $\$ 0.015$ & $\$ 0.013$ & $\$ 0.012$ & $\$ 0.010$ \\
\hline
\end{tabular}

Source: ICF analysis based on equipment manufacturer input.

Figura 5: Costos WHP. Fuente: ICF International, sección 3.3 de costos, página 20.

Esta información facilitó la proyección de los costos de capital, teniendo en cuenta la dificultad de encontrar los precios en dólares de las plantas de biomasa.

Esta tabla plasma el costo en USD / KW de operación y mantenimiento, calculada como un porcentaje sobre el costo de capital, ubicada en el módulo de "Costos". 


\section{Mercado}

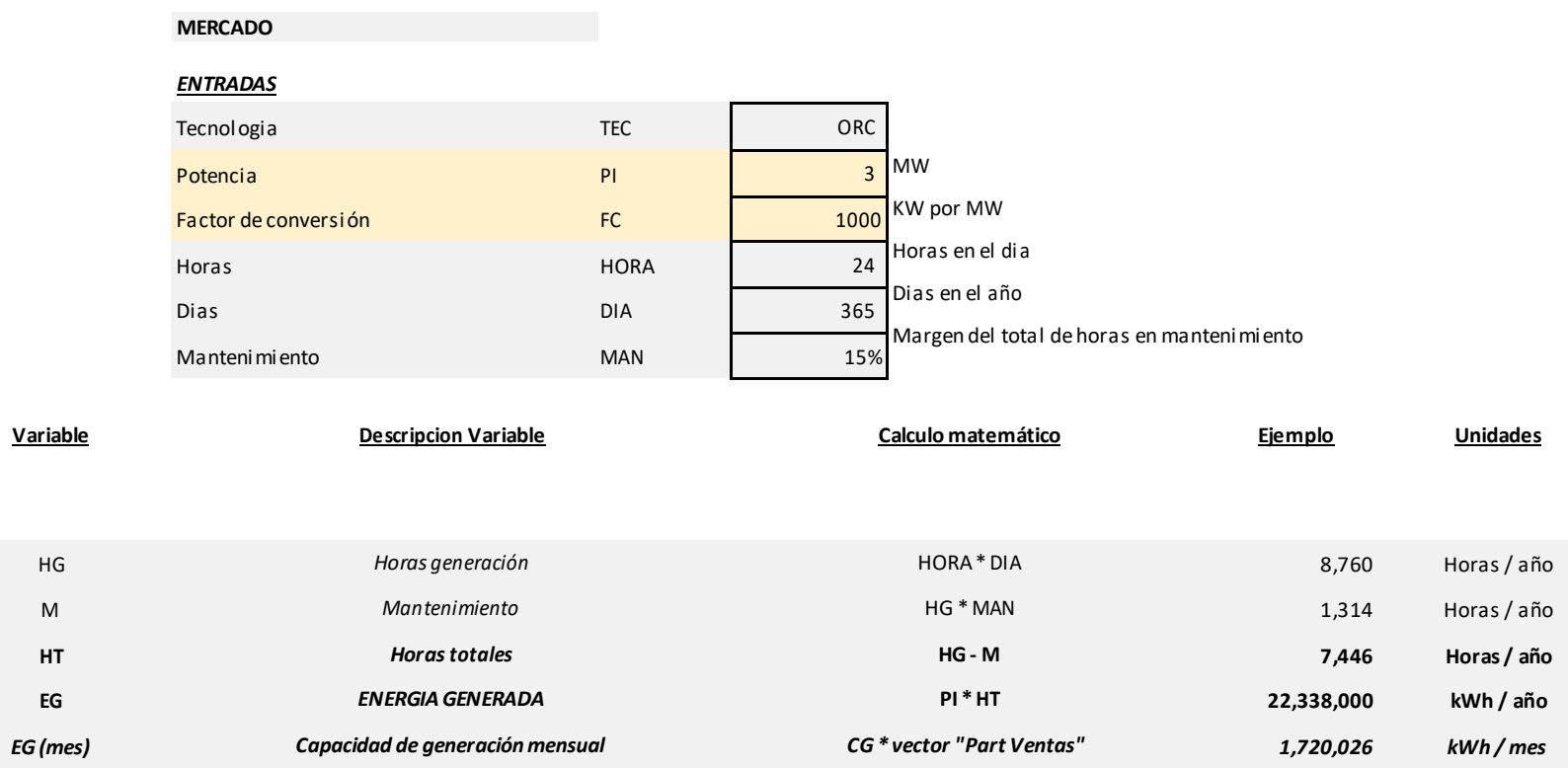

Figura 6: Modulo Mercado. Elaboración propia.

En este módulo se calcularon las horas totales de generación de energía, horas de mantenimiento, y la capacidad de generación en $\mathrm{kWh}$ al año. Las variables se encuentran ordenadas de tal manera que algunas entradas provienten del modulo de inversión inicial junto con las suministradas por el usuario.

Con una potencia instalada de $3 \mathrm{MW}$, la energia generada del proyecto es de $22.338 .000 \mathrm{kWh}$ al año. En el flujo de caja, se va a crear un vector denominado "Participación" el cual pondera un porcentaje por mes, para que el usuario visualice la generación energética acorde a su preferencia. 


\begin{tabular}{|cc|}
\hline \multicolumn{2}{|c|}{ Vector "Part. Ventas" } \\
Mes 1 & $7.70 \%$ \\
Mes 2 & $7.70 \%$ \\
Mes 3 & $8.10 \%$ \\
Mes 4 & $8.20 \%$ \\
Mes 5 & $8.30 \%$ \\
Mes 6 & $8.40 \%$ \\
Mes 7 & $8.50 \%$ \\
Mes 8 & $8.50 \%$ \\
Mes 9 & $8.50 \%$ \\
Mes 10 & $8.60 \%$ \\
Mes 11 & $8.70 \%$ \\
Mes 12 & $8.80 \%$ \\
TOTAL & $100.00 \%$ \\
\hline
\end{tabular}

*: Al multiplicar por CG genera una matriz y se deben incorporar el numero de años del proyecto

Figura 7: Vector "participacion ventas". Elaboración propia.

Estas variables permitirán generar la proyección de ingresos. 


\section{Ingresos}

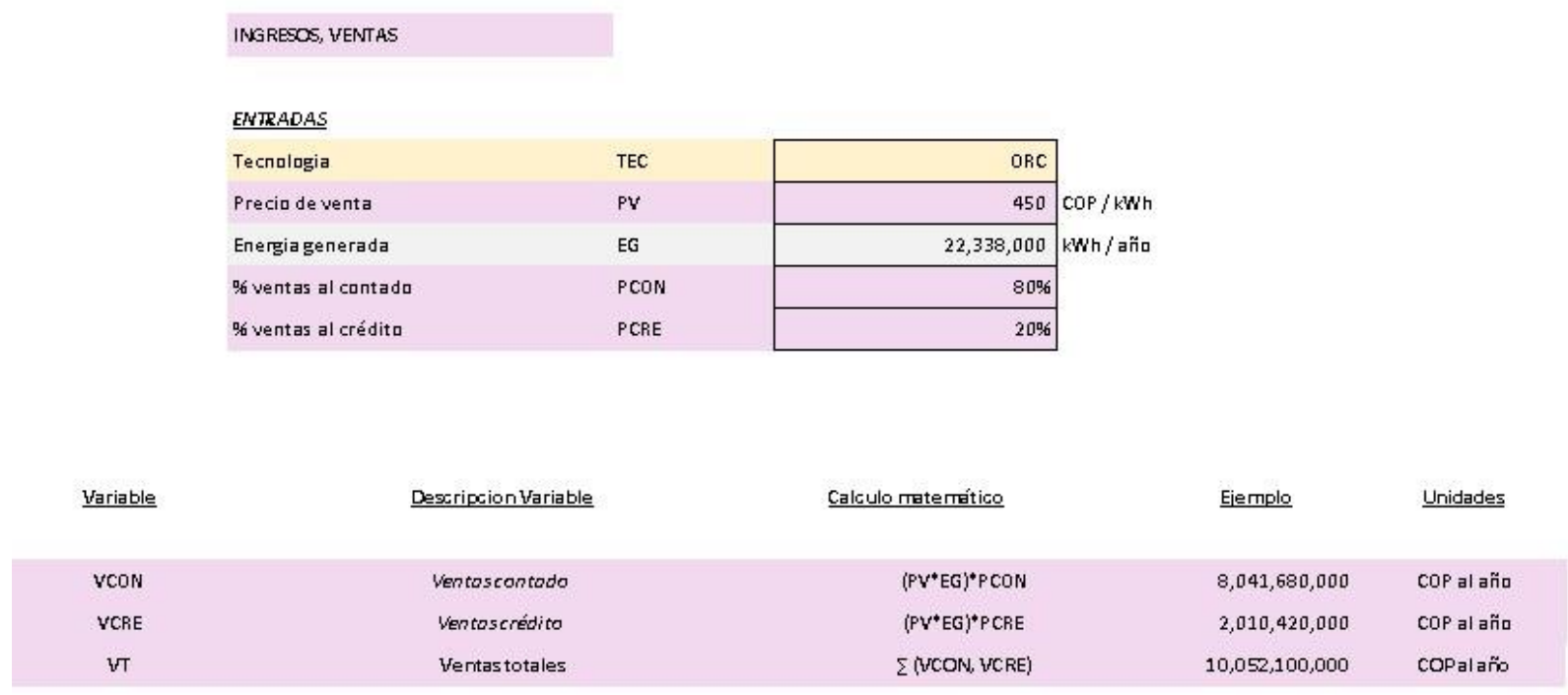

Precia de venta de $\$ 450$ pes as par $\mathrm{kWh}$

Figura 8: Modulo ingresos, ventas. Elaboración propia.

Este modulo calcula el ingreso en pesos colombianos a partir de la tecnologìa ORC , variable del módulo “A. INVERSIÓN INICIAL” y de EG, del módulo “mercado”, junto con las entradas suministradas por el usuario (precio de venta y \% ventas).

Se procede a calcular el ingreso por tipo de cliente y las ventas totales, con un precio de venta estimado de \$ $450 \mathrm{COP} / \mathrm{kWh}$. 


\subsection{Costo nivelado}

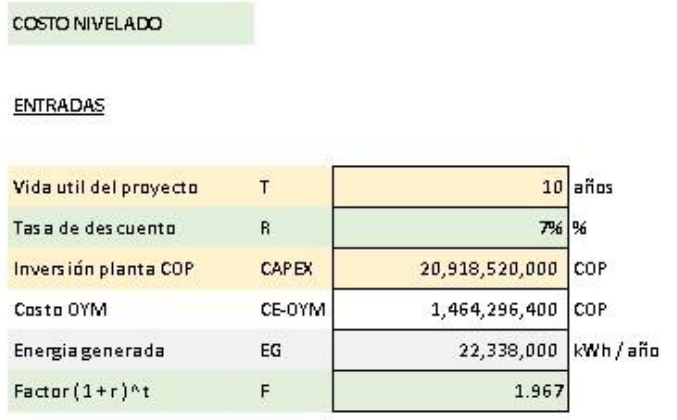

\begin{tabular}{|c|c|c|}
\hline T & 10 & \\
\hline $\mathrm{R}$ & 706 & \\
\hline CAPEX & $20,918,520,000$ & \\
\hline CE-OYM & $1,464,296,400$ & \\
\hline EG & $22,338,000$ & \\
\hline $\mathrm{F}$ & 1.967 & \\
\hline
\end{tabular}

Variable

LCOE
Descripcion Variable

Costa Nivelada (levelized cas ts)
Calculo matemático

$\frac{C A P E X+\frac{\sum(C E-O Y M \times T)}{F}}{\frac{E G \times T}{F}}$
Eiemplo

$\$ 249.77$
Unidades

$\operatorname{cop} / \mathrm{kWh}$

Praducir cada kWhs ale a 250 pes as

Figura 9: Modulo “costo nivelado”. Elaboración propia.

Este módulo involucra variables definidas en A. INVERSIÓN INICIAL y en el módulo "mercado", así como la tasa de descuento suministrada por el usuario. El costo de operación y mantenimiento corresponde al 7\% sobre el CAPEX al año.

El costo nivelado calcula el valor en COP / kWh, utilizado para comparar en estas unidades el costo de generación de cada tecnologia. El resultado obtenido arroja un costo de generación de \$250 pesos por kWh tomando como referencia los valores estimados. 


\subsection{Costos y gastos}

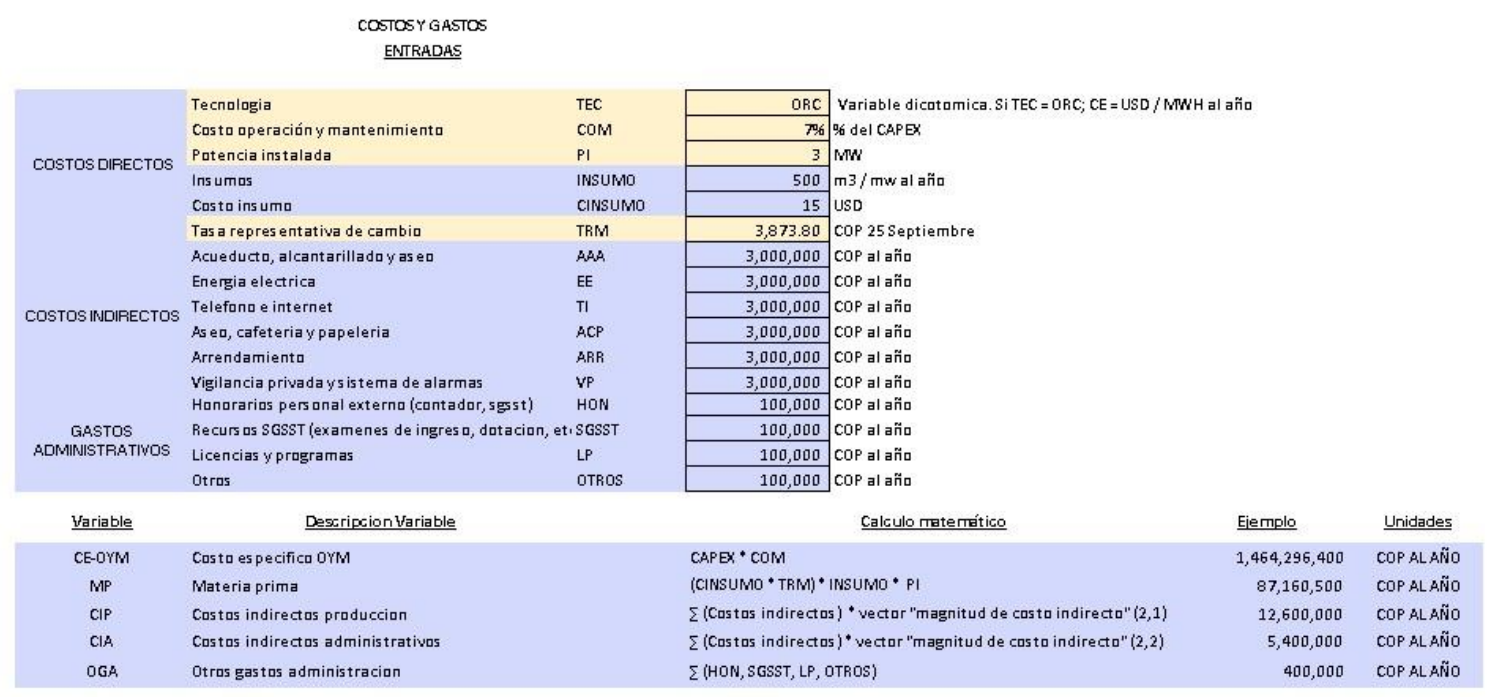

Figura 10: Modulo "costos y gastos". Elaboración propia.

Este módulo contiene los costos y gastos del proyecto, divididos por área. La estructura organizacional se compone de producción y administracion, cuyo funcionamiento implica el gasto de recursos, definidos a partir de presupuestos anuales. La distinción de costo contra gasto se realiza para su ubicación en el estado de resultados:

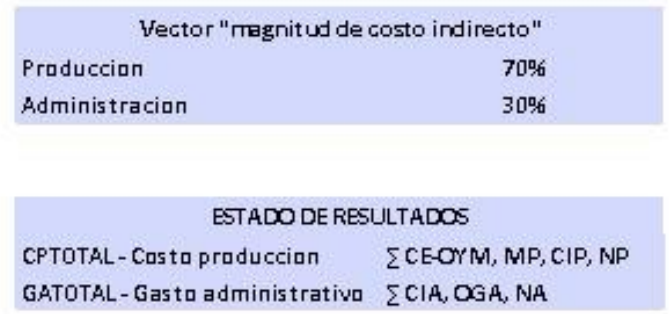

Figura 11: Vectores CYG. Elaboración propia

A partir del presente vector, el usuario puede definir la magnitud de costo indirecto que corresponde a cada área. 


\section{Nomina}
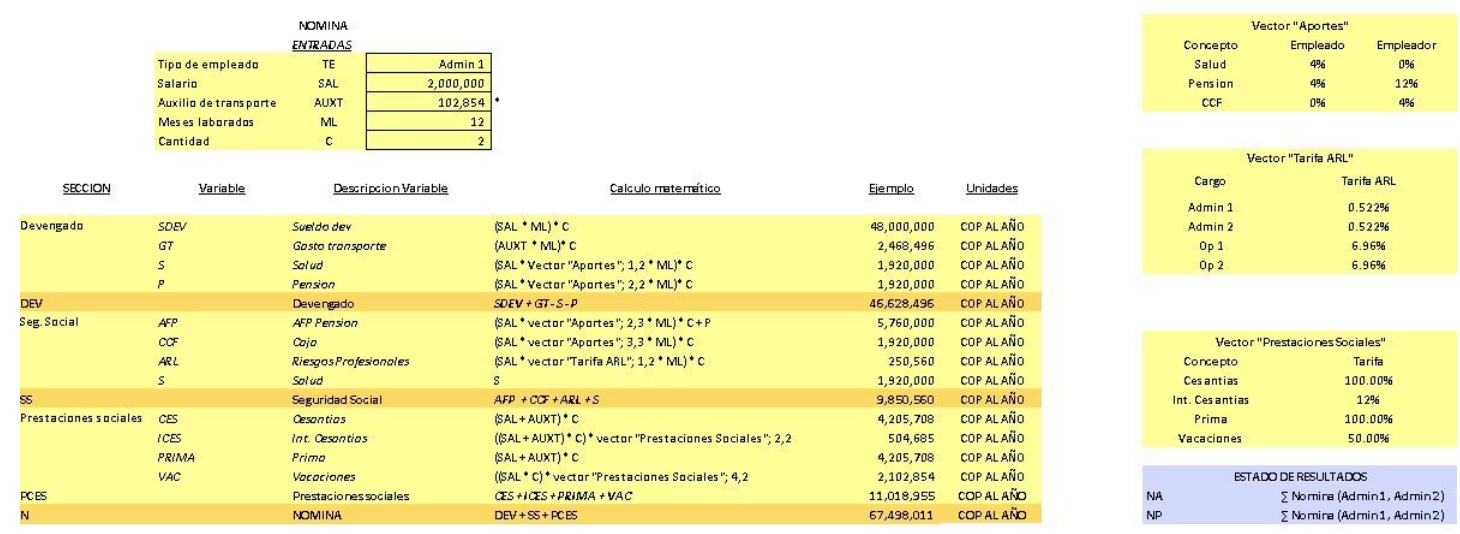

Figura 11: Modulo “nómina”. Elaboración propia

Este modulo agrupa los salarios, deducciones realizadas, gastos de seguridad social y prestaciones sociales. El usuario selecciona el tipo de empleado y la cantidad para calcular los valores que dependiendo del área, se clasifican entre costo y gasto. Esto tambien se relaciona con la tarifa de ARL a pagar, puesto que para el personal de producción se debe pagar el 6.96\% de su salario por desempeñar actividades de alto riesgo (V), a diferencia del personal administrativo que cotiza sobre el riesgo minimo (I). 


\subsection{Financiación}

\begin{tabular}{l} 
GASTOS NO OPERACIONALES \\
5,1: Financiacion \\
\begin{tabular}{l|r|} 
ENTRADAS \\
\hline Monto \\
TEA (Tasa Efectiva Anual) \\
Plazo años \\
Plazo meses
\end{tabular}$\quad 1.091 .852 .000$ \\
\hline
\end{tabular}

\begin{tabular}{|c|c|c|}
\hline Variable & Descripcion Variable & Calculo matemático \\
\hline TEM & Tasa efectiva mensual & $(1+\text { Tasa Efectiva Anual })^{\wedge}(1 / 12)-1$ \\
\hline c & Cuota & $\left(\right.$ Monto $*\left(T E M \times(1+\text { TEM })^{\wedge} n\right) /\left((1+\text { TEM })^{\wedge} n\right)-1$ \\
\hline INT & Interes & Monto*TEM \\
\hline CAP & Capital & Cuota -interes \\
\hline $\mathrm{Fl}$ & Financiacion total & $\Sigma(C A P, I N T)$ \\
\hline s & Saldo & Monto-capital \\
\hline \multicolumn{3}{|c|}{ te la misma operacion de calcular intereses y capital durante los 60 meses que dure el crédito. } \\
\hline \multicolumn{3}{|c|}{ I flujo de caja y ER se considera gasto no operacional } \\
\hline \multicolumn{2}{|c|}{ CAP TOTAL } & $\sum$ (matriz "financiacion" casilla capital) \\
\hline \multicolumn{2}{|l|}{ INT TOTAL } & $\sum$ (matriz"financiacion" intereses) \\
\hline
\end{tabular}

$\begin{array}{rc}\frac{\text { Ejemplo }}{1,389 \%} & \frac{\text { Unidades }}{96} \\ 34.281 .997,15 & \text { COP MENSUAL } \\ 29.052 .540,80 & \text { COP MENSUAL } \\ 5.229 .456,34 & \text { COP MENSUAL } \\ 411.383 .966 & \text { COP AL AÑO } \\ 2.086 .622 .544 & \text { COP MENSUAL . }\end{array}$

Figura 12: Modulo "financiación". Elaboración propia

En este módulo el usuario ingresa el monto a solicitar a la entidad financiera, la tasa efectiva anual y el plazo, para calcular la tasa efectiva mensual, cuota, intereses y el saldo.

La utilidad de este modulo aporta a que el usuario pueda ver el costo total de intereses por pagar a la entidad financiera, para escoger la mas adecuada a su estructura de costos, y optimizar el recurso económico.

Este rubro hace parte de los gastos no operacionales. 


\subsection{Depreciación}

GASTOS NO OPERACIONALES

5.2 Depreciacion
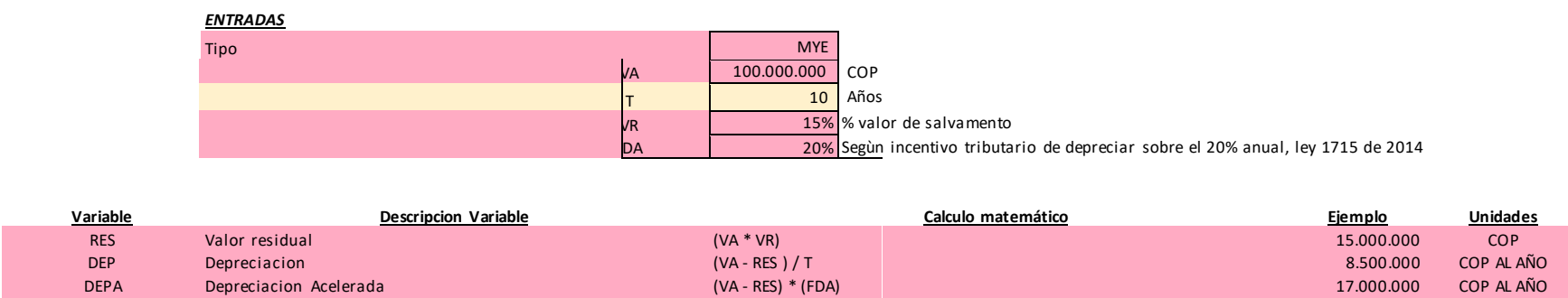

Figura 13: Modulo “depreciación”. Elaboración propia

Este modulo incorpora la depreciación de activos en linea recta, y la depreciación acelerada, en la que la inversion en maquinaria para proyectos FNCER puedan amortizarse a un valor máximo del 20\% anual, como incentivo tributario de la ley 1715 de 2014.

El usuario determina si su activo tiene valor residual en caso que quiera venderlo, reconociendo su valor en el proceso contable. Con esto, se calcula el valor a depreciar sobre la vida util del activo. 


\section{Impuestos}

IMPUESTOS

ENTRADAS

Ventas totales

Vida util del proyecto

Inversion planta

Tarifa ICA

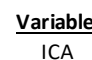

INC INV

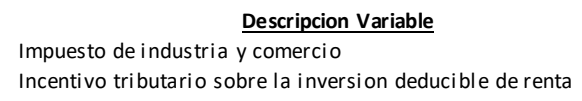

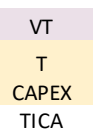

\begin{tabular}{|c|}
\hline 10.052 .100 .000 \\
\hline 10 \\
\hline 20.918 .520 .000 \\
\hline $11,04 / 1000$ \\
\hline
\end{tabular}

$\mathrm{VT} * 11,04 / 1000$ (CAPEX * 50\%) / T

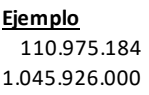

$\underline{\text { Unidades }}$

COP AL AÑO COP AL AÑO

Figura 14: Modulo “impuestos”. Elaboración propia

Este modulo calcula los impuestos a pagar cuya base son las ventas totales. La generación de energía eléctrica está gravada con la tarifa ICA del 11.04/1000, para el calculo del ICA anual.

Asimismo, se calcula el incentivo tributario por la inversión en proyectos FNCER deducible del impuesto a la renta, correspondiente al 50\% del CAPEX dividido durante la vida util del proyecto.

El impuesto a la renta no se calcula en este módulo puesto que la base es la utilidad antes de impuestos. 


\section{Resultados}

ESTADO DE RESULTADOS DEL PROYECTO

\begin{tabular}{|c|c|c|c|c|c|c|c|c|c|c|c|c|c|}
\hline \multirow{2}{*}{\multicolumn{2}{|c|}{ Tasa de descuento }} & \multirow[b]{3}{*}{ SIGNO } & \multirow[t]{2}{*}{$7 \%$} & \multirow[b]{2}{*}{ Anualidad } & & \multirow[b]{3}{*}{3} & \multirow[b]{3}{*}{4} & \multirow[b]{3}{*}{5} & \multirow[b]{3}{*}{6} & \multirow[b]{3}{*}{7} & \multirow[b]{3}{*}{8} & \multirow[b]{3}{*}{9} & \multirow[b]{3}{*}{10} \\
\hline & & & & & & & & & & & & & \\
\hline RUBRO & CONCEPTO & & Variable & 1 & 2 & & & & & & & & \\
\hline & Ventas & + & VT & 10.052 .100 .000 & 10.052 .100 .000 & 10.052.100.000 & 10.052 .100 .000 & 10.052 .100 .000 & 10.052 .100 .000 & 10.052.100.000 & 10.052 .100 .000 & 10.052 .100 .000 & 10.052 .100 .000 \\
\hline OPERATIVOS & Costos & - & CPTOTAL & 1.564 .056 .900 & 1.564 .056 .900 & 1.564 .056 .900 & 1.564.056.900 & 1.564.056.900 & 1.564.056.900 & 1.564 .056 .900 & 1.564.056.900 & 1.564 .056 .900 & 1.564 .056 .900 \\
\hline & Gastos & - & GATOTAL & 73.298.011 & 73.298 .011 & 73.298.011 & 73.298.011 & 73.298.011 & 73.298.011 & 73.298.011 & 73.298.011 & 73.298.011 & 73.298 .011 \\
\hline \multirow{11}{*}{ NO OPERATIVOS } & Financiacion & - & $\mathrm{FI}$ & 411.383.966 & 411.383.966 & 411.383 .966 & 411.383 .966 & 411.383.966 & 411.383.966 & 411.383.966 & 411.383.966 & 411.383.966 & 411.383.966 \\
\hline & ICA Anual & - & ICA & 110.975 .184 & 110.975 .184 & 110.975 .184 & 110.975 .184 & 110.975 .184 & 110.975 .184 & 110.975 .184 & 110.975 .184 & 110.975 .184 & 110.975 .184 \\
\hline & Depreciacion & - & DEP & 17.000 .000 & 17.000 .000 & 17.000 .000 & 17.000 .000 & 17.000 .000 & 17.000 .000 & 17.000 .000 & 17.000 .000 & 17.000 .000 & 17.000 .000 \\
\hline & Inversión inicial & - & CAPEX & 20.918 .520 .000 & & & & & & & & & \\
\hline & Impuestos (renta 33\%) & - & & & 2.635.499.171 & 2.635.499.171 & 2.635.499.171 & 2.635.499.171 & 2.635.499.171 & 2.635.499.171 & 2.635.499.171 & 2.635.499.171 & 2.635 .499 .171 \\
\hline & MENOS: INC INV & + & & 1.045 .926 .000 & 1.045.926.000 & 1.045 .926 .000 & 1.045.926.000 & 1.045.926.000 & 1.045 .926 .000 & 1.045.926.000 & 1.045.926.000 & 1.045.926.000 & 1.045 .926 .000 \\
\hline & utilidad & & & 11.997.208.061 & 6.285 .812 .769 & 6.285 .812 .769 & 6.285.812.769 & 6.285.812.769 & 6.285.812.769 & 6.285.812.769 & 6.285.812.769 & 6.285.812.769 & 6.285.812.769 \\
\hline & VPN & & 27.061.983.176,9 & & & & & & & & & & \\
\hline & TIR & & $51 \%$ & & \multicolumn{2}{|c|}{ Proyecto viable, pdte calcular impuestos } & & & & & & & \\
\hline & \multicolumn{6}{|c|}{ Inversion / utilidad año 1} & & & & & & & \\
\hline & Payback & & & $\begin{array}{r}3,33 \\
39,93\end{array}$ & $\begin{array}{l}\text { Años para } \\
\text { Meses p }\end{array}$ & $\begin{array}{l}\text { mo de inversión } \\
\text { etomo inversion }\end{array}$ & & & & & & & \\
\hline
\end{tabular}

Precio de venta

LCOE * TIR

Figura 15: Estado de resultados. Elaboración propia

Las variables generadas en los modulos anteriores permitirán construir el estado de resultados del proyecto, y los indicadores financieros: Valor Presente Neto, Tasa Interna de Retorno, Payback, y precio de venta en el que se incorpora la TIR hallada. La columna "signos" muestra la estructura matemática, cuyo calculo final es la utilidad del ejercicio. Con los valores calculados se estima que la inversión se recuperará en 3,3 años, con un precio de venta de 377 pesos por kWh, VPN positiva y TIR del 52\%, siendo viable el proyecto en estas condiciones. 


\section{Conclusiones}

- El proceso anteriormente descrito plasma un orden sistemático en la generación de variables que le permite al usuario entender paso a paso la estructura del cada rubro del balance, asi como del estado final de resultados y su lógica matemática.

- Este ejercicio se puede implementar para cualquier tipo de tecnologia y trasladarse a lenguajes de programación de tal manera que el usuario solamente deba insertar las entradas solicitadas y genere los informes que requiera, toda vez que el usuario tenga conocimiento previo de los presupuestos que se van a ejecutar, asi como de los datos de orden técnico que guardan relacion con la producción de energía.

- El estado de resultados generado resulta versatil en el sentido que resume la información mas relevante para la toma de decisiones, y la parametrización da la libertad de configurar y visualizar diferentes informes de series de tiempo a solicitud del usuario, tales como flujo de caja y efectivo, balance general, entre otros.

- La presente herramienta debe estar soportada del estudio tecnico previo para su respectiva implementación.

BIOTECH \& ENGINEERING Untels. Jul - Dic.1(2), 2021; ISSN:2788 -4295; 86-110 


\section{Referencias}

AENE Consultoria S.A - UPME. (2003). Potencialidades de los cultivos energéticos y residuos agrícolas en Colombia: Informe final. Recuperado el 25 de Mayo de 2020, de Unidad de Planeación Minera y Energética: https://bdigital.upme.gov.co/handle/001/1287

Food and Agriculture Organization for the United Nations FAO. (2014). El planteamiento sobre bioenergía y seguridad alimentaria - BEFS de la FAO. Guía de implementación.

Recuperado el 30 de Mayo de 2020, de http://www.fao.org/3/a-i3672s.pdf

ICF INTERNATIONAL. (March de 2015). Waste heat to power market assessment. Recuperado el 22 de Septiembre de 2020, de https://info.ornl.gov/sites/publications/Files/Pub52953.pdf

Mesa Sierra, J. (2016). Condiciones de trabajo en dos empresas del sector forestal, ubicadas en los departamentos de Cundinamarca y Amazonas. Enero-Julio 2016. Recuperado el 1 de Junio de 2020, de Universidad Nacional de Colombia, :

http://bdigital.unal.edu.co/57412/1/35479767.2017.pdf

Ministerio de Ambiente y Desarrollo Sostenible. (2015). Uso y legalidad de la madera en Colombia. Análisis parcial. Recuperado el 25 de Mayo de 2020, de https://www.minambiente.gov.co/images/BosquesBiodiversidadyServiciosEcosistemicos/ pdf/Gobernanza_forestal_2/12._Uso_y_Legalidad_de_la_Madera.pdf

Ministerio Federal Alemán de Alimentación y Agricultura BMEL \& Food and Agriculture Organization for the United Nations FAO. (Febrero de 2014). Bioenergía y Seguridad Alimentaria, evaluación rápida BEFS RA. Componente madera combustible y residuos de madera. Sección 2: Presupuesto para plantaciones de madera como combustible. 